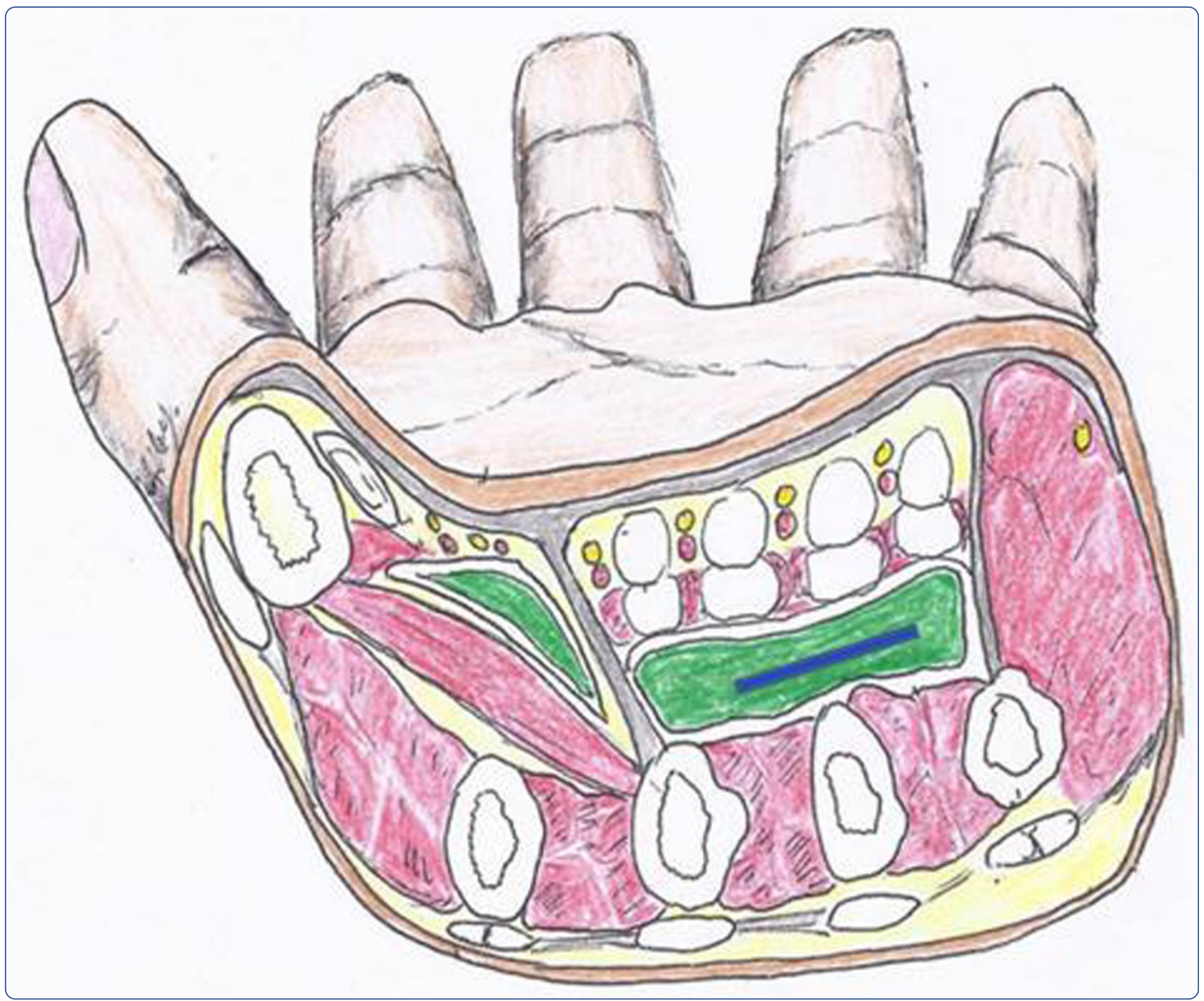

Retained palmar foreign body presenting as a late hand infection: proposed diagnostic algorithm to detect radiolucent objects Ipaktchi et al. 


\title{
Retained palmar foreign body presenting as a late hand infection: proposed diagnostic algorithm to detect radiolucent objects
}

Kyros Ipaktchi ${ }^{i^{*}}$, Andrew DeMars ${ }^{3}$, Jung Park ${ }^{1}$, Christopher Ciarallo ${ }^{2}$, Meryl Livermore ${ }^{1}$ and Rodrigo Banegas ${ }^{1}$

\begin{abstract}
Background: Penetrating injuries to the hand can compromise important anatomic structures, and persisting foreign objects may become a source of infection. Foreign body intrusions into the hand are among the most common injuries to the upper extremity seen in the Emergency Department. Radiolucent organic objects, as well as a few higher density inorganic materials such as plastic, present a diagnostic challenge and are routinely missed using standard radiography. While the literature describes the use of high-frequency ultrasound as an adjunct to conventional diagnostics, to our knowledge, no formal algorithm has been published.

Case presentation: We describe a case of incomplete wooden splinter removal, presenting as a late midpalmar abscess five months after the initial injury, and requiring two subsequent surgical explorations for definitive treatment. This case has led us to implement a formal diagnostic pathway including high-frequency ultrasound at our institution. We contrast this presentation with a subsequent case involving a much smaller wooden palmar foreign body that was easily identified under ultrasound and removed without sequelae.

Conclusion: Many hand injuries are caused by low density, radiolucent foreign bodies. These objects can easily escape traditional evaluation in the emergency room including standard radiography. We present an algorithm implementing high frequency ultrasound to minimize the risk of missing radiolucent penetrating foreign objects in the hand.
\end{abstract}

Keywords: Foreign objects, Midpalmar space, Ultrasound

\section{Background}

Hand injuries are among the most frequent causes for Emergency Department visits, many of which are due to foreign body intrusion [1]. The density of important anatomic structures explains the increased rate of complex injuries and long-term morbidity. Standard physical hand examination fails to detect up to $38 \%$ of foreign bodies, and routine radiographs identify wooden objects in only $15 \%$ of cases [2,3]. Overall detection of foreign bodies of any material by plain film radiography has been quoted at $80 \%$ [4]. In contrast, high-frequency ultrasound ( $\geq 7.5 \mathrm{MHz}$ ) has been shown to identify foreign bodies with a sensitivity of $87-93 \%$ and a specificity of $89-99 \%$ [5]. Failure to detect organic foreign bodies

\footnotetext{
* Correspondence: Kyros.ipaktchi@dhha.org

${ }^{1}$ Department of Orthopaedic Surgery, Denver Health Medical Center, 777 Bannock Street, Denver, CO 80204, USA

Full list of author information is available at the end of the article
}

can lead to infection and functional morbidity. Plant thorns are of particular concern since they have low structural density and high radiolucency, and persisting thorns may lead to chronic synovitis [6].

\section{Case presentation}

Case \# 1

A 39 year-old right hand dominant male, presented to an outside facility after a large wooden splinter had pierced through the hypothenar eminence of his nondominant hand and had broken off. Per report, a splinter was removed and plain film radiography was unremarkable. However, despite the procedure, the patient reported a persisting density in his palm. Eight weeks later, the patient presented to our Emergency Department complaining of a "popping" sensation, palmar erythema and swelling in the affected hand. Clinically, the hand was neurovascular intact, with intact tendon 


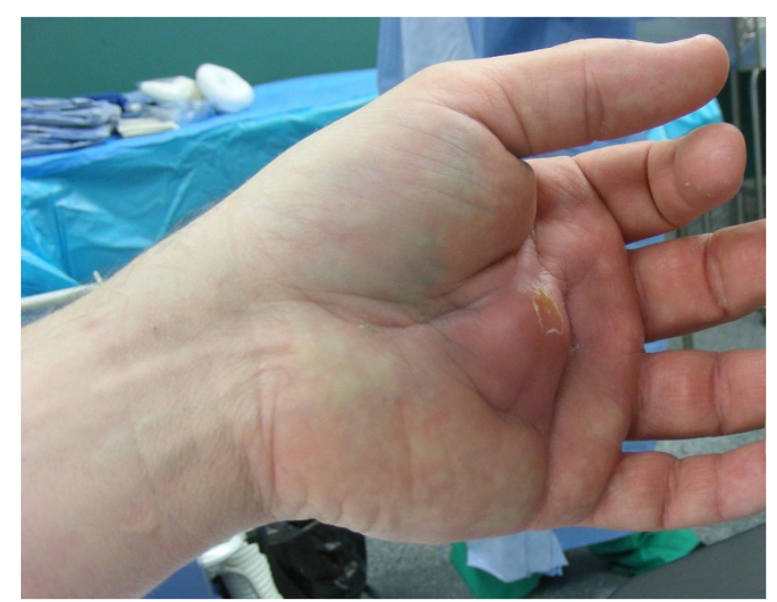

Figure 1 Intraoperative photo showing a deep palmar abscess. Five months after incomplete wooden splinter removal the patient is readmitted for surgical exploration. Fingers are held in protective flexion position due to compartmental swelling of the deep palmar space.

function. Forced passive extension was painful in the palm and there was obvious local swelling. The patient had a mild leukocytosis, while C-reactive protein and sedimentation rates were normal. Plain film radiographs were unremarkable except for a well-healed fifth metacarpal fracture and the presence of palmar soft tissue swelling.

The patient was taken to the operating room for irrigation and debridement. Exploration of his palm revealed no foreign body or purulence. However, there was inflammation involving the flexor tendon sheath. After an uneventful recovery, the patient regained full finger function and resumed work. Three months later, the patient returned to the Emergency Department with

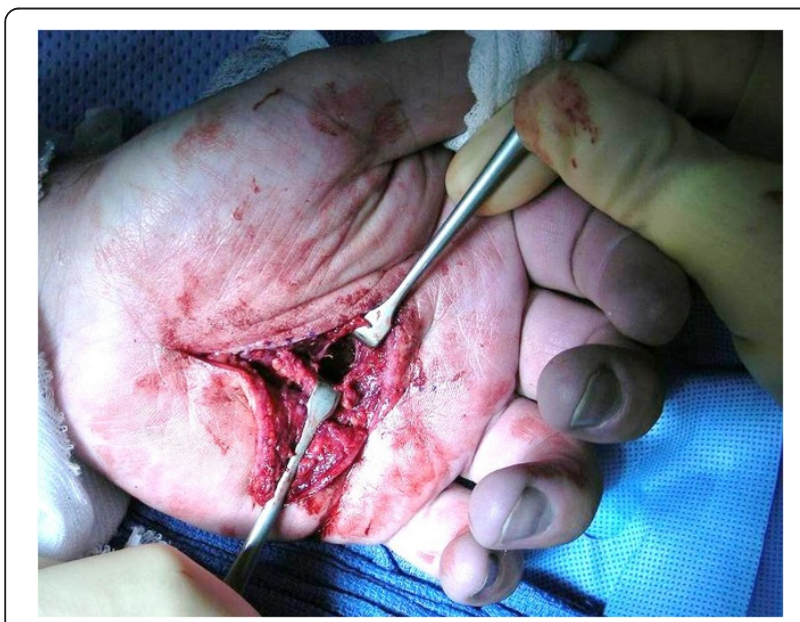

Figure 2 Opened deep palmar space. Surgical exploration of the mid-palmar space abscess which had decompressed through the flexor tendons and palmar aponeurosis.

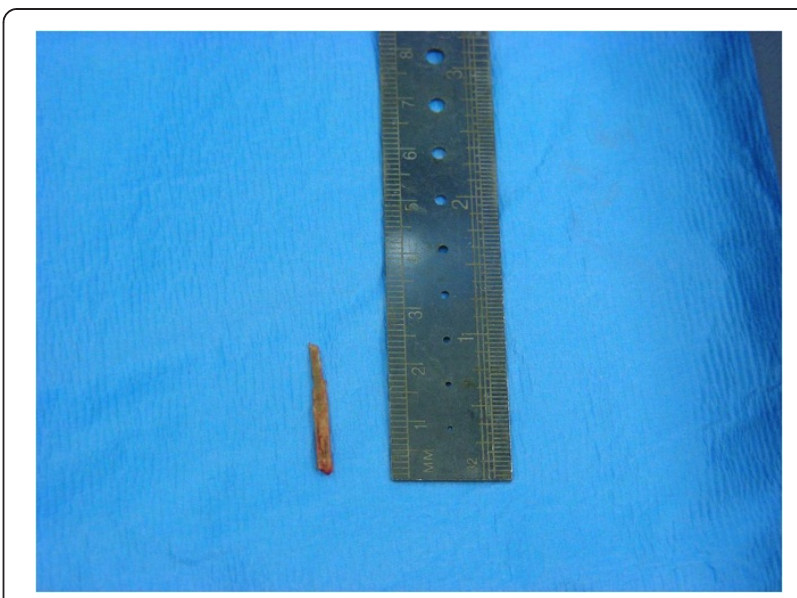

Figure 3 Retrieved radiolucent one-inch long wooden splinter. The operation yielded the surprising finding of a near 1 inch long wooden splinter which had resided for 5 months undetected in the deep palmar space.

a fluctuant abscess in the affected palm (Figure 1). Surgical exploration at that time showed a large epifascial abscess, which originated in the mid-palmar space and had spread superficially (Figure 2). Further deep exploration revealed, surprisingly, a retained one-inch long wooden splinter (Figure 3). The deep location of the splinter below the flexor tendons in the midpalmar space may have contributed to the failed detection of this object during the first exploration (Figure 4). Subsequent recovery after removal of the foreign body was uneventful.

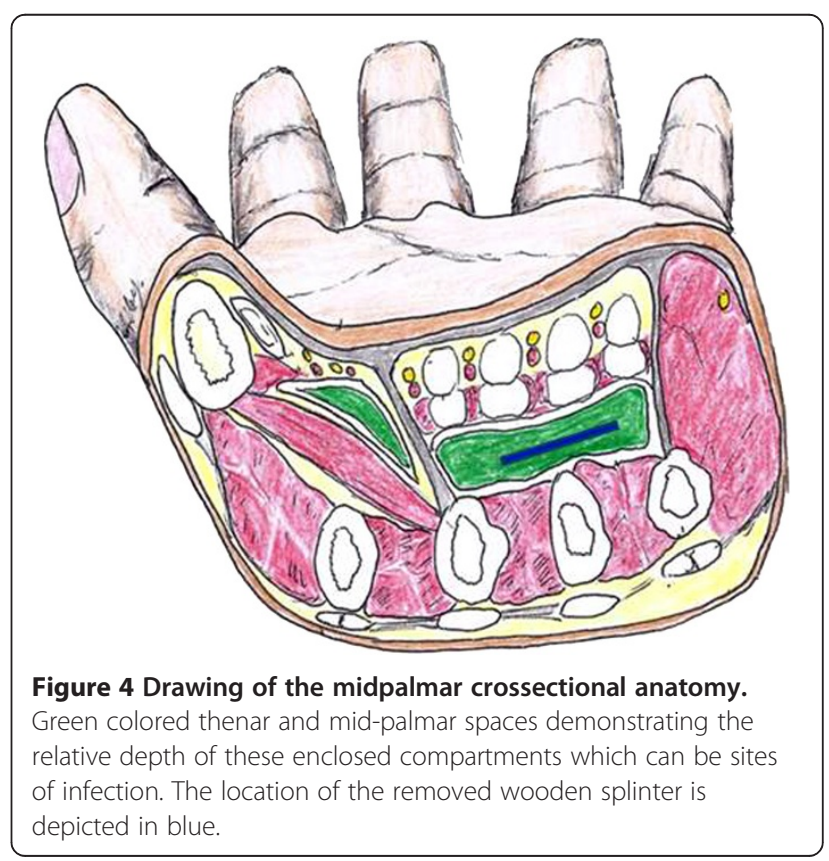




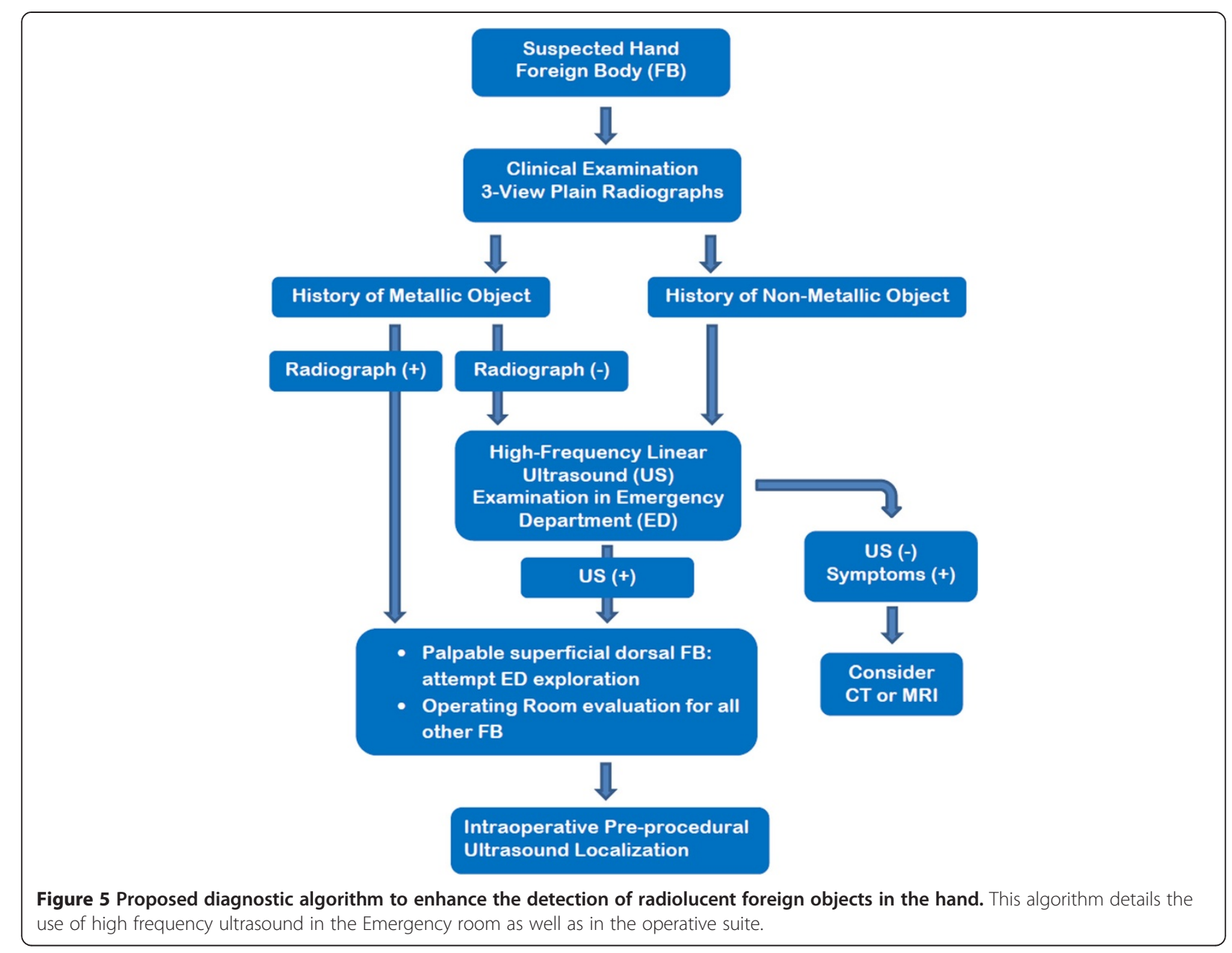

\section{Case \# 2}

A 46 year-old carpenter had sustained a wooden splinter injury six months before presenting in hand clinic with tenderness in the first webspace. There were no signs of infection on clinical exam and plain film radiographs were negative. Our new foreign body diagnostic pathway was implemented and the high-frequency ultrasound examination revealed a $2.7 \mathrm{~mm}$ foreign object in the first webspace (Figures 5 and 6). Subsequent surgical exploration revealed a retained wooden splinter, and the patient had an uneventful recovery.

\section{Discussion}

Common low-density organic materials - such as thorns, wood and fish bones - along with some higher density inorganic materials - such as glass and plastic - account for a large percentage of hand foreign bodies. Historically, soft tissue radiography has been described as a useful diagnostic adjunct for foreign body localization [7]. However, as radiodensity is a consequence of both material density and

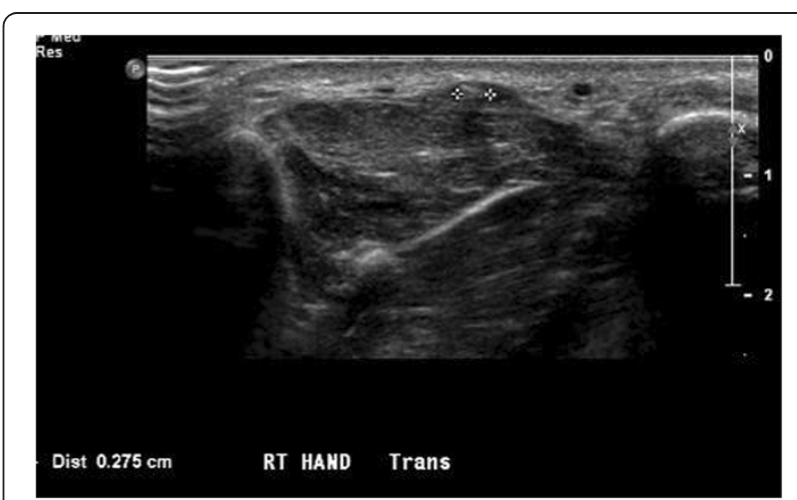

Figure 6 High frequency linear ultrasound image. Ultrasound detection of a $2.7 \mathrm{~mm}$ wooden splinter in the first webspace distance measurement in between the 2 tracking cross marks. This demonstrates the resolution of current ultrasound probes which would have allowed for easy detection of the nearly $3 \mathrm{~cm}$ or 1 inch long splinter shown in Figure 3. 
atomic number, wood is consistently elusive on plain film radiography [2].

Soon after its clinical introduction, computerized tomography (CT) was found to be a highly useful modality to detect deep and embedded low density objects [8]. Although computerized tomography is readily available in most hospitals, it is typically not performed as a dynamic study, and the exposure to ionizing radiation is significant. Magnetic resonance imaging (MRI) has also been advocated for soft tissue foreign body localization. However, there are significant financial costs and long acquisition times, and extremity MRI protocols are frequently limited to two millimeter section intervals. Additionally, magnetic resonance imaging may produce an unacceptable number of false-positive diagnoses, as structures such as scar tissue, tendons and calcifications may be mistaken for foreign bodies [9].

Ultrasound has been used for foreign body localization since 1978 [10]. While the ultimate resolution limit of a $10 \mathrm{MHz}$ sound wave in tissue is approximately $0.15 \mathrm{~mm}$, practical application of high-frequency ultrasound has identified foreign objects as small as $0.5 \mathrm{~mm}$ and as deep as $4 \mathrm{~cm}$ [3]. The use of ultrasound conducting gel as a standoff pad or immersing the extremity in a water bath during visualization have both been described to improve the detection of superficial foreign bodies and to minimize patient discomfort from direct transducer pressure [11]. Dynamic ultrasound scanning should be performed, and the tissue should be imaged in two planes in an axis perpendicular to the surface of the suspected foreign body.

Most foreign bodies are hyperechoic, and the induced inflammatory changes appear as a surrounding hypoechoic rim, beginning within twenty-four hours of intrusion [5]. Metal and glass may cause a "comet tail" artifact, while gravel demonstrates strong posterior acoustic shadowing [11]. Power Doppler may be used to identify acute inflammatory changes and neogranulation around foreign bodies, but it may not be positive for up to two days after intrusion [12]. Limitations to ultrasound detection of soft tissue foreign bodies include gas bubbles, hematomas, calcified soft tissues and operator inexperience. While some authors have argued that high-frequency ultrasound should be the imaging modality of choice for foreign body localization, others have suggested that ultrasound should replace conventional fluoroscopy for preoperative localization [3].

\section{Conclusion}

Many hand injuries are caused by low density, radiolucent foreign bodies. Superficial foreign bodies can frequently be localized during physical examination and removed without difficulty. However, radiolucent objects, particularly those that are smaller and deeper, may not be identified on plain film radiographs or even during superficial surgical exploration. As compared to other soft tissue imaging modalities such as CT and MRI, high-frequency ultrasound appears to be an equally efficacious, more costeffective, and readily available option in most Emergency Departments. Additionally, high-frequency ultrasound allows for a dynamic, real-time intervention with concurrent evaluation of adjacent soft tissues. With the intent to optimize foreign body detection, minimize cost and reduce exposure to ionizing radiation, we present a formal diagnostic algorithm that includes ultrasound to evaluate suspected hand foreign bodies.

\section{Consent}

Written informed consent was obtained from the patient for publication of this Case report and any accompanying images. A copy of the written consent is available for review by the Editor-in-Chief of this journal.

\section{Abbreviations}

MHz: Megahertz; Mm: Millimeter; CT: Computerized tomography; MRI: Magnetic resonance imaging; US: Ultrasound.

\section{Competing interests}

The authors declare that they have no competing interests.

\section{Authors' contribution}

$\mathrm{KI}$ provided the case and idea and prepared the manuscript, $\mathrm{AD}$ and JP reviewed the manuscript, CC reviewed of manuscript and provided the ultrasound content and designed Figure 6 together with Kl; RB reviewed the manuscript and drew Figure 4, ML reviewed the manuscript. All authors read and approved the final manuscript.

\section{Author details}

${ }^{1}$ Department of Orthopaedic Surgery, Denver Health Medical Center, 777 Bannock Street, Denver, CO 80204, USA. ²Department of Anesthesiology, Denver Health Medical Center, 777 Bannock Street, Denver, CO 80204, USA. ${ }^{3}$ University of Colorado School of Medicine, Anschutz Campus, Aurora, CO 80045, USA.

Received: 24 May 2013 Accepted: 5 July 2013

Published: 11 July 2013

\section{References}

1. Frazier WH, Miller M, Fox RS, Brand D, Finseth F: Hand injuries: incidence and epidemiology in an emergency service. JACEP 1978, 7:265-268.

2. Anderson MA, Newmeyer WL 3rd, Kilgore ES Jr: Diagnosis and treatment of retained foreign bodies in the hand. Am J Surg 1982, 144:63-67.

3. Banerjee B, Das RK: Sonographic detection of foreign bodies of the extremities. Br J Radiol 1991, 64:107-112.

4. Donaldson JS: Radiographic imaging of foreign bodies in the hand. Hand Clin 1991, 7:125-134.

5. Boyse TD, Fessell DP, Jacobson JA, Lin J, van Holsbeeck MT, Hayes CW: US of soft-tissue foreign bodies and associated complications with surgical correlation. Radiographics 2001, 21:1251-1256.

6. Tung CH, Chen YH, Lan HH, Hsieh TY, Chen DY, Lan JL: Diagnosis of plantthorn synovitis by high-resolution ultrasonography: a case report and literature review. Clin Rheumatol 2007, 26:849-851.

7. Mucci B, Stenhouse G: Soft tissue radiography for wooden foreign bodies-a worthwhile exercise? Injury 1985, 16:402-404.

8. Bauer AR Jr, Yutani D: Computed tomographic localization of wooden foreign bodies in children's extremities. Arch Surg 1983, 118:1084-1086.

9. Khanna S, Crues JV 3rd: Complexities of MRI and false positive findings. Ann N Y Acad Sci 2009, 1154:239-258.

10. Hassani SN, Bard RL: Real time ophthalmic ultrasonography. Radio/ 1978, 127:213-219. 
11. Tirado A, Wu T, Noble VE, Huang C, Lewiss RE, Martin JA, Murphy MC, Sivitz A: Ultrasound-guided procedures in the emergency departmentdiagnostic and therapeutic asset. Emerg Med Clin North Am 2013, 31:117-149.

12. Davae KC, Sofka CM, DiCarlo E, Adler RS: Value of power Doppler imaging and the hypoechoic halo in the sonographic detection of foreign bodies: correlation with histopathologic findings. J Ultrasound Med 2003, 22:1309-1313. quiz 1314-1306.

doi:10.1186/1754-9493-7-25

Cite this article as: Ipaktchi et al:: Retained palmar foreign body presenting as a late hand infection: proposed diagnostic algorithm to detect radiolucent objects. Patient Safety in Surgery 2013 7:25.

\section{Submit your next manuscript to BioMed Central and take full advantage of:}

- Convenient online submission

- Thorough peer review

- No space constraints or color figure charges

- Immediate publication on acceptance

- Inclusion in PubMed, CAS, Scopus and Google Scholar

- Research which is freely available for redistribution 